\title{
Effect of micro-osteoperforations on external apical root resorption: A randomized controlled trial
}

\section{Azaitun Akma Shahrin (1) Sarah Haniza Abdul Ghani Noraina Hafizan Norman (1)}

Centre of Paediatric Dentistry and Orthodontic Studies, Faculty of Dentistry, Universiti Teknologi MARA, Jalan Hospital, Sungai Buloh, Malaysia
Objective: This study aimed to investigate the effect of micro-osteoperforations (MOPs) on external apical root resorption (EARR) during the initial orthodontic alignment phase of maxillary anterior crowding. Methods: Thirty patients (25 females, 5 males; mean age, $22.66 \pm 3.27$ years) who presented with moderate crowding of the upper labial segment and underwent extraction-based fixed appliance treatment were recruited. They were randomly allocated to receive adjunctive therapy with MOPs $(n=15)$ or treatment with fixed appliances only (control group; $n=15$ ). EARR was measured from long-cone periapical radiographs taken at the start and the sixth month of treatment. A correction factor for the enlargement difference was used to calculate EARR. Data were analyzed with descriptive statistics and repeated-measures analysis of variance. Results: The mean root lengths of 168 teeth were measured and showed no statistically significant difference $(p>0.05)$ after six months of fixed appliance treatment in the MOP (mean difference $[\mathrm{MD}]=0.13 \mathrm{~mm}$; 95\% confidence interval $[\mathrm{Cl}]=-0.10-0.35)$ and control group $(\mathrm{MD}=0.14 \mathrm{~mm} ; 95 \% \mathrm{Cl}=$ $-0.10-0.37)$. Most of the roots in the MOP and control groups $(42.86 \%$ and $52.38 \%$, respectively) showed only mild resorption. Less than $8 \%$ of the roots in both groups (7.14\% in the MOP group and $4.76 \%$ in the control group) showed moderate resorption. Conclusions: Acceleration of orthodontic tooth movement with adjunctive MOPs therapy during the alignment phase does not exacerbate EARR in patients with moderate crowding of the upper labial segment in comparison with controls.

[Korean J Orthod 2021;51(2):86-94]

Key words: Micro-osteoperforations, External apical root resorption, Orthodontic alignment, latrogenic effect

Received June 9, 2020; Revised October 20, 2020; Accepted October 23, 2020.

Corresponding author: Noraina Hafizan Norman.

Lecturer, Centre of Paediatric Dentistry and Orthodontic Studies, Faculty of Dentistry, Universiti Teknologi MARA, Jalan Hospital, 47000 Sungai Buloh, Malaysia.

Tel +60-124914707 e-mail noraina@uitm.edu.my

How to cite this article: Shahrin AA, Abdul Ghani SH, Norman NH. Effect of microosteoperforations on external apical root resorption: A randomized controlled trial. Korean J Orthod 2021;51:86-94.

(C) 2021 The Korean Association of Orthodontists.

This is an Open Access article distributed under the terms of the Creative Commons Attribution Non-Commercial License (http://creativecommons.org/licenses/by-nc/4.0) which permits unrestricted non-commercial use, distribution, and reproduction in any medium, provided the original work is properly cited. 


\section{INTRODUCTION}

External apical root resorption (EARR) is one of the iatrogenic effects of fixed appliance treatment. Resorption activity during orthodontic tooth movement (OTM) is associated with a wide range of cytokines and molecular factors that are involved in clastic cell fusion and activation, including receptor activator of nuclear factor kappa-B ligand (RANKL)/RANK/osteoprotegerin (OPG) and interleukin (IL)-1-related pathways.' The OPG:RANKL ratio in the crevicular fluid varies according to the magnitude of the compressive force. Root resorption is initiated by macrophage-like cells from the periodontal ligament blood supply, causing damage to the nearby cementoblast layer overlying the cementoid. This leads to cementum exposure, causing the denuded root surface to become more susceptible to resorption by scavenger cells and osteoclasts. ${ }^{1}$

Many factors contribute to EARR, and they can be categorized as patient- and treatment-related factors. Biological elements include genetic susceptibility (IL$1 \beta$ gene), systemic disease, age, sex, previous resorption due to trauma, personal habits, and tooth structure. ${ }^{2}$ However, a recent meta-analysis suggested that the IL$1 \beta$ polymorphism is not correlated with a predilection to EARR. ${ }^{3}$ Earlier literature showed that the incidence and severity of root resorption was connected with numerous mechanical factors, including force magnitude, the amount or distance of tooth movement, force type, and treatment appliance. ${ }^{4,5}$ Increased treatment time or prolonged treatment duration positively correlated with increased EARR ${ }^{4}$; thus, shortening the treatment duration is a worthwhile goal as it could decrease the risk of EARR.

Various surgical techniques ranging from total block osteotomies to flapless partial corticotomy have been reported to accelerate OTM with different degrees of invasiveness. ${ }^{6}$ Corticotomy involves superficial punctures or cuts on the cortical bone. It is considered invasive since it would necessitate the use of full-thickness mucoperiosteal flaps either buccally or lingually, followed by suturing. ${ }^{7} \mathrm{Al}-\mathrm{Naoum}$ et al. ${ }^{7}$ reported in their splitmouth study that alveolar corticotomy increases canine retraction by 2- to 4 -fold on the experimental side than on the control side. However, this procedure compromises the dental periodontium and is associated with surgical risks such as an increase in postoperative pain, discomfort, and swelling. ${ }^{8}$ Due to its high morbidity, this technique has low acceptance among patients. Since then, corticotomy surgical procedures have evolved into flapless techniques.

Piezocision is a flapless corticotomy technique in which a microsurgical blade is used to make gingival microincisions, which are followed by piezoelectric inci- sions of the cortical alveolar bone made with an ultrasonic piezosurgical knife. ${ }^{9,10}$ Charavet et al. ${ }^{11}$ reported that treatment length was significantly shortened by $43 \%$ in the piezocision group compared to that in the conventional orthodontic treatment, without affecting gingival recession, bone dehiscence, and root resorption. Another part of their study reported that this technique was associated with a higher degree of apprehension and pain in the trial group. ${ }^{10}$ Even though piezocision is less invasive and has been reported to be effective in accelerating $\mathrm{OTM},{ }^{12}$ the procedure involves the potential risk of scarring. ${ }^{11,13}$

The use of micro-osteoperforations (MOPs) is the most current minimally invasive modality to accelerate OTM; MOPs have better acceptance among patients, and their application is more favorable than other invasive adjunctive methods in routine practice. However, a single application of MOPs does not accelerate OTM, considering the rate of canine or en masse retraction. ${ }^{14}$ The first study of the effect of MOPs on the rate of OTM in humans by Alikhani et al. ${ }^{15}$ did not investigate the associated root resorption due to the short duration of only one month. In their study, dental panoramic tomography (DPT) showed no indisputable evidence of root resorption or alveolar bone loss. However, this technique was considered to be not precise enough for measuring the magnitude of root resorption; thus, further studies are warranted. Subsequently, several randomized controlled trials (RCTs) have been conducted to investigate the effects of MOPs on EARR. Sivarajan et al. ${ }^{14}$ included eight RCTs in their systematic review, and only two RCTs reported EARR as the secondary outcome while evaluating the effects of MOPs on canine retraction. The authors concluded that MOPs do not have an effect on root resorption.

Thus, root resorption related to MOPs has not been studied extensively, with the reported studies primarily focusing on root resorption during space closure. As a result, additional studies investigating the association between MOPs and root resorption are warranted. This study aimed to investigate the effect of MOPs on EARR during the six-month initial orthodontic alignment phase of anterior maxillary crowding. The secondary outcome was to assess the frequency of EARR according to severity in relation to the MOP procedure.

\section{MATERIALS AND METHODS}

\section{Ethics approval}

Ethical approval for this study was obtained by the Universiti Teknologi MARA (UiTM) Research Ethics Committee on September 29, 2017 (Reference: 600-IRMI [5/1/6]; REC/297/17). This trial was also registered retrospectively at the International Standard Randomised 
Controlled Trial Number (ISRCTN) registry with the study identification (ID) ISRCTN15080404. Participation was voluntary, and withdrawal from the trial was allowed without jeopardizing the patient's orthodontic treatment.

\section{Trial design}

This study was a prospective, randomized, singlecenter, two-arm parallel clinical trial to compare the effects of MOPs on EARR during the initial alignment stage of moderate maxillary crowding in comparison with conventional orthodontic treatment alone. The trial was conducted at the Orthodontic Clinic, Faculty of Dentistry, UiTM.

\section{Participants}

Inclusion criteria included age ranging from 18 to 45 years, moderate crowding of $5-8 \mathrm{~mm}$ in the maxillary arch, full dentition up to the second molars prior to extraction of the upper first premolars with or without anchorage reinforcement, healthy periodontal status with probing depth less than or equal to $3 \mathrm{~mm}$, full-mouth plaque score of $20 \%$ or lower, and full-mouth bleeding score of $20 \%$ or lower. Exclusion criteria included previous orthodontic treatment, systemic diseases, smoking, pregnancy, and compromised periodontal oral health with evidence of bone loss and root resorption on DPT, as well as history of dental trauma. Patients with dental (e.g., hyperdontia, hypodontia) or craniofacial anomalies (e.g., cleft lip and palate) were also excluded. Patients receiving medications that could interfere with tooth movement, such as anti-inflammatory drugs, systemic corticosteroids, or calcium channel blockers were not taken into consideration. The period of recruitment was from October 2017 until February 2019.

\section{Orthodontic treatment}

Standardized conventional pre-adjusted edgewise McLaughlin, Bennett, and Trevisi brackets with a 0.022 $\times 0.028$-inch (in) slot (Victory Series; 3M Unitek, Monrovia, CA, USA) were used for all participants. The archwire sequence was similar for both groups, which involved the use of 0.014-in nickel titanium (NiTi) archwires followed by 0.018-in NiTi archwires (TruFlex NiTi archwires; Ortho Technology, Lutz, FL, USA) until alignment was achieved.

\section{Micro-osteoperforation procedure}

A single operator (A.A.S.) performed MOPs under local anesthesia infiltration (Lignocaine with 1:100,000 adrenaline). The patients in the MOP group received two perforations $3 \mathrm{~mm}$ apart within the attached gingiva to $1 \mathrm{~mm}$ apical to the mucogingival junction, with the MOPs placed equidistant between the anterior teeth from the upper right canine to the upper left canine, except in the midline alveolar bone. The perforations were $1.5-\mathrm{mm}$ wide and 3- $\mathrm{mm}$ deep and were made using a disposable MOP device (PROPEL Ortho Singapore, Kallang, Singapore). All participants underwent followup assessments at four-week intervals, and MOPs were repeated at every visit until completion of the alignment stage. Postoperatively, local measures with cotton roll pressure were applied to arrest bleeding after MOPs. Participants in the MOP group were prescribed 0.12\% chlorhexidine digluconate (Oradex; CAVICO, Selangor, Malaysia) mouthwash to be used twice a day for one week. In addition, proper oral hygiene maintenance was reinforced, and the participants were advised to avoid modifying their flossing and brushing routines in the MOP region.

\section{Measurement of root resorption}

Long-cone intraoral periapical (1OPA) radiographs were used to assess the occurrence of EARR. All images were taken by a standardized paralleling technique using a single digital dental intraoral machine (Satelec X-MindAC/DC; Acteon, VA, ltaly). 1OPA radiographs involving six anterior maxillary teeth were taken before bracket placement, on initiation of MOPs (T1), and after six months into treatment (T2) (Figure 1). Crown length and root length were measured using a virtual ruler of the EasyDent V4 viewer software (Vatech, Hwaseong, Korea) by identifying these landmarks in all three radiographs at each stage (Figure 2). The crown length was gauged from the mid-incisal point of the crown to the median cementoenamel junction (CEJ) point, which was the midpoint between the mesial and distal CEJ. The root length was calculated from the median CEJ point to the tipping point of the root. Apical root resorption was measured as the difference between the length of the root at T1 and the length of the root at T2 in millimeters.

Image magnification between pre- and post-observational radiographs was rectified by exploiting the crown length as a reference, assuming crown lengths to be unaffected throughout the trial interval. Consequently, a correction factor (CF) was formulated by dividing the initial crown length (C1) by the post-treatment crown length (C2). The CF was computed to relate the pretreatment (R1) and post-treatment (R2) root length of each tooth, as shown in the following equations. Then, the EARR per tooth was calculated in millimeters using the formula adapted from previous studies ${ }^{16,17}$ :

$$
\begin{gathered}
C F=C 1 / C 2 \\
\text { EARR }=R 1-(R 2 \times C F)
\end{gathered}
$$

The grading of apical root resorption was assessed 


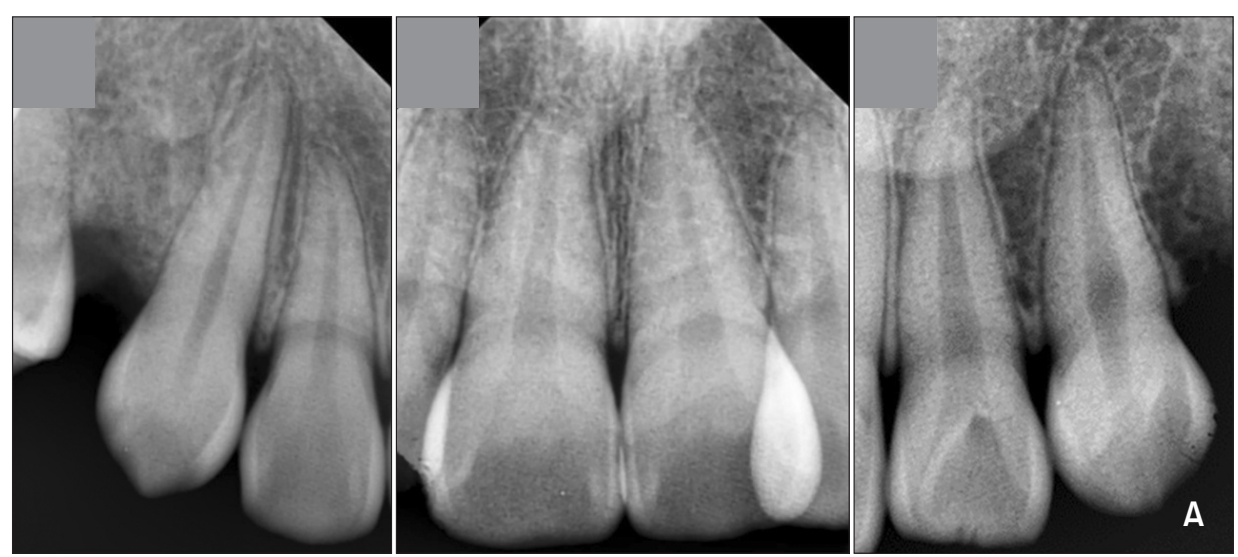

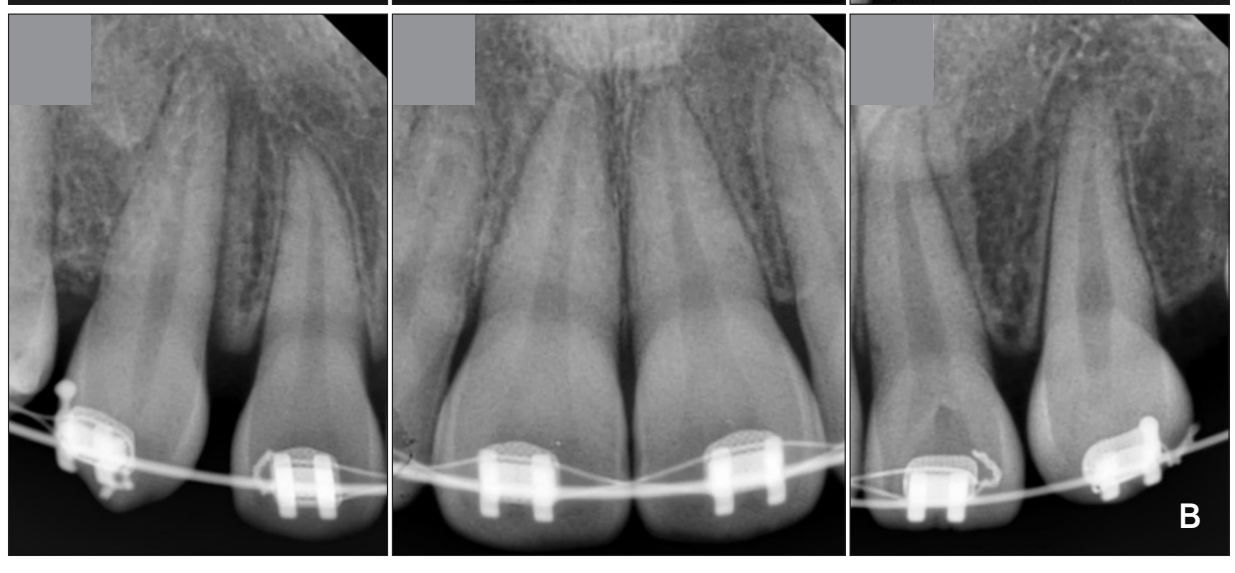

Figure 1. Intraoral periapical (IOPA) radiographs. A, PreIOPA taken before orthodontic treatment. B, Post-IOPA repeated after 6 months of treatment.
A

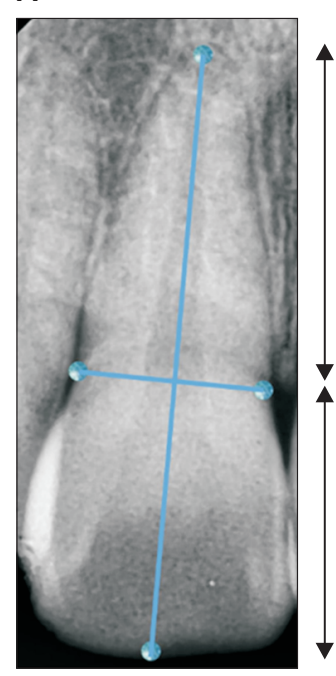

B

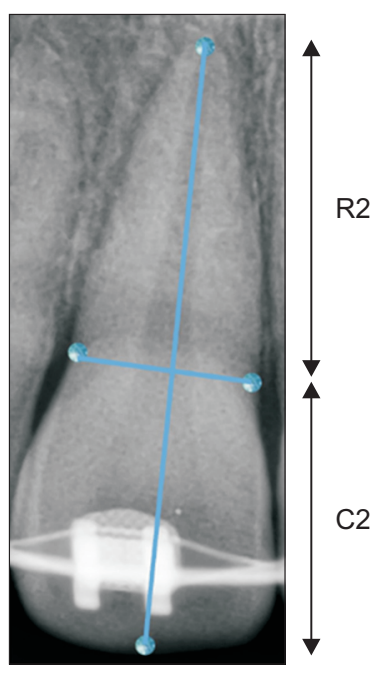

Figure 2. Measurements of total root and crown length at (A) T1: pre-treatment, (B) T2: post-treatment after 6 months were performed on intraoral periapical radiographs at the median point of the tooth axis.

R1, pre-treatment root length; R2, post-treatment root length; $\mathrm{C} 1$, pre-treatment crown length; $\mathrm{C} 2$, post-treatment crown length.

separately by the principal investigator (A.A.S.) in the 10PA images by adapting the scoring system proposed by Levander and Malmgren. ${ }^{18}$

\section{Sample size calculation}

The sample size was calculated to detect a significant mean difference of $0.54 \mathrm{~mm}$ of root resorption with a standard deviation of $0.47 \mathrm{~mm}$ during the alignment stage of fixed appliance treatment after six months. ${ }^{19}$ The statistical power was set at $80 \%$, with a confidence interval $(\mathrm{Cl})$ of 95\%. A minimum of 12 participants for each group was required, and this number was increased to 15 per group considering a $20 \%$ dropout rate.

\section{Randomization}

Once the participant agreed to the study protocol, written informed consent was obtained. Participants were allocated randomly to the MOP or control group in a 1:1 allocation ratio using block randomization of six numbers (three odd and three even numbers). Odd numbers were allocated to the MOP group while even numbers were assigned to the control group. Sequentially numbered opaque, sealed envelopes were used for concealed randomization. Cards were written with numbers and set in opaque sealed envelopes, which were kept by the central trial coordinator (N.H.N.), who was respon- 
sible for assigning the envelope numbers upon operator request (A.A.S.). The subjects' participation protocol is shown in the Consolidated Standards of Reporting Trials flow diagram (Figure 3).

\section{Blinding}

Blinding of participants and the operator was not feasible due to the nature of the intervention. Thus, the outcome examiner was blinded to the subject's assigned intervention. Patient information was concealed by the central trial coordinator (N.H.N.) prior to the measurement assessments.

\section{Statistical analysis}

Descriptive and analytical statistics were obtained using the SPSS software version 25.0 (IBM Corp., Armonk, NY, USA). The statistical significance level was prespecified at $p<0.05$, with a $95 \% \mathrm{Cl}$. Significant changes in EARR were analyzed at the patient level using repeated-measures analysis of variance to compare the mean differences in EARR among groups. Stata software version 13 (StataCorp, College Station, TX, USA) was used for kappa analysis.

Calibration for root resorption was performed on 15 IOPA radiographs to ensure the reliability of the measurement technique. The intra-rater reliability was excellent with an intra-class correlation coefficient of 0.82 , with the $95 \% \mathrm{Cl}(0.65,0.91)$, indicating a low level of random error. Further analysis with a paired ttest revealed no significant difference between the two readings $(p=0.242)$. Grading for the extent of EARR was also performed with an interval of two weeks on 15 IOPA radiographs, and the intra-rater reliability of Cohen's kappa was 0.880 with $93.33 \%$ agreement $(p<$ 0.001).

\section{RESULTS}

\section{Baseline characteristics}

A total of 35 participants were eligible for this trial. However, five participants were excluded, including three patients who declined to participate and two who had moved away. The remaining 30 participants who met the inclusion criteria were enrolled for the trial. Subsequently, one pregnant participant dropped out from the MOP group, and one participant in the control group was lost with no follow-up records. An equal number of participants in both groups was maintained throughout the observation period. Thus, analysis as per protocol was undertaken to compare root resorption between the MOP and control groups. The mean age in the MOP and control groups was $22.80 \pm 3.78$ years and 22.50 \pm 2.74 years, respectively, with no significant difference between the groups $(p=0.810)$ (Table 1$)$.

\section{Root resorption}

A comparison of the changes in root length (Table 2) showed no significant difference between the MOP and control groups ( $p=0.238$ and $p=0.225$, respectively). Further analysis between groups also did not show a

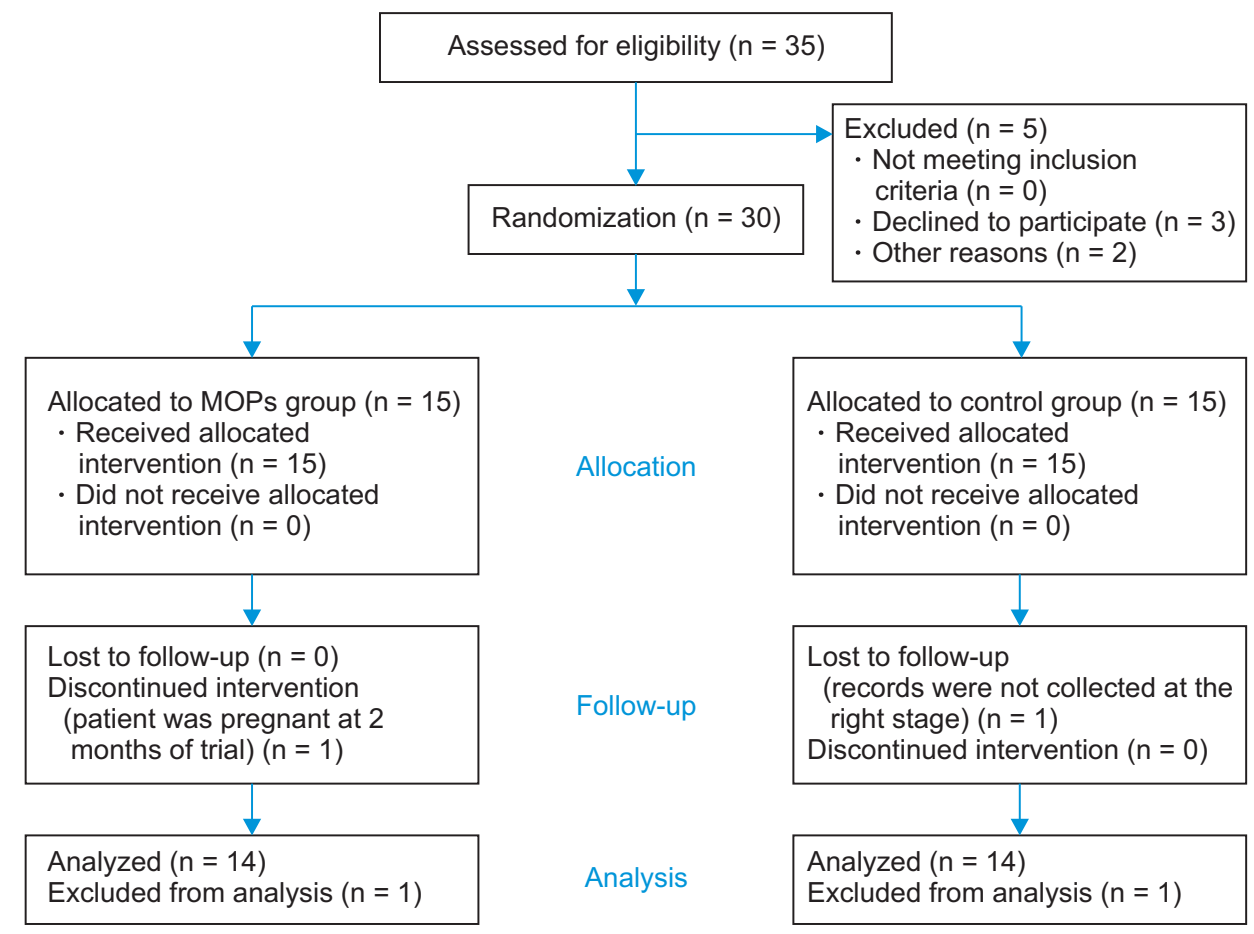

Figure 3. Consolidated Standards of Reporting Trials (CONSORT) study flow diagram. MOP, micro-osteoperforation. 
Table 1. Demographic characteristics of the participants

\begin{tabular}{llccc}
\hline \multicolumn{1}{c}{ Variable } & & MOPs $(\mathbf{n}=\mathbf{1 5})$ & Control $(\mathbf{n}=\mathbf{1 5})$ & $\boldsymbol{p}$-value \\
\hline Age $(\mathrm{yr})$ & & $22.80 \pm 3.78$ & $22.50 \pm 2.74$ & $0.810^{*}$ \\
Sex & Male & $2(13.3)$ & $3(20.0)$ & $>0.950^{\dagger}$ \\
& Female & $13(86.7)$ & $12(80.0)$ & \\
Incisor relationship & Class I & $6(40.0)$ & $9(60.0)$ & $0.564^{\dagger}$ \\
& Class II/1 & $4(26.7)$ & $3(20.0)$ & \\
& Class II/2 & $2(13.3)$ & $0(0)$ & \\
& Class III & $3(20.0)$ & $3(20.0)$ & \\
\hline
\end{tabular}

Values are presented as mean \pm standard deviation or number (\%).

MOPs, micro-osteoperforations.

*Independent $t$-test was performed.

${ }^{\dagger}$ Fisher's exact test was performed.

Table 2. Intergroup comparison of change in the root length $(\mathrm{mm})$ between groups in six months duration

\begin{tabular}{lccccc}
\hline \multirow{2}{*}{ Group } & \multicolumn{4}{c}{ Within-group } \\
\cline { 2 - 5 } & Pre-treatment & Post-treatment & Mean difference (95\% CI) & $\boldsymbol{t}$ statistics (df) & $\boldsymbol{p}$-value \\
\hline MOP $(\mathrm{n}=14)$ & $9.17 \pm 0.62$ & $9.04 \pm 0.57$ & $0.13(-0.10,0.35)$ & $1.23(14)$ & 0.238 \\
Control $(\mathrm{n}=14)$ & $9.38 \pm 0.74$ & $9.24 \pm 0.77$ & $0.14(-0.10,0.37)$ & $1.28(13)$ & 0.225 \\
\hline
\end{tabular}

Values are presented as mean \pm standard deviation.

$\mathrm{CI}$, confidence interval; MOP, micro-osteoperforation; n, number of participants.

Repeated-measures analysis of variance (ANOVA) within-group analysis. Significance level $(\alpha)=0.05$. Repeated-measures ANOVA between-group analysis reveals no significant difference in root resorption $(p=0.419)$ between the MOP and control groups.

significant difference $(p=0.419)$.

Approximately $50 \%$ of the teeth in both groups showed no root resorption in the first six months. Most of the roots in the MOP and control groups $(42.86 \%$ and $52.38 \%$, respectively) showed only mild resorption. Less than $8 \%$ of the roots in both groups $(7.14 \%$ in the MOP group and $4.76 \%$ in the control group) showed moderate resorption (Table 3 ).

\section{DISCUSSION}

\section{Prevalence of external apical root resorption}

Mild resorption is defined as some alteration of the root apex, while moderate resorption indicates less than $2 \mathrm{~mm}$ of root resorption. ${ }^{18} \mathrm{In}$ an extensive study for developing an index for root resorption with an observation period of six to nine months after orthodontic treatment, Levander and Malmgren ${ }^{18}$ reported that $66 \%$ of the teeth showed zero or minor resorption, $33 \%$ showed moderate resorption, 17\% showed resorption, and 1\% showed extreme resorption. Similarly, in their study of 302 patients, Smale et al. ${ }^{20}$ assessed root resorption for 4 incisors over 6 months after initial orthodontic alignment and reported that $47.1 \%$ of the patients showed no root resorption, $29.9 \%$ and $19.4 \%$
Table 3. Frequency of root resorption according to severity between groups (percentage)

\begin{tabular}{clcl}
\hline Grade & Severity & MOPs & Control \\
\hline 0 & No & $42(50.00)$ & $36(42.86)$ \\
1 & Mild & $36(42.86)$ & $44(52.38)$ \\
2 & Moderate & $6(7.14)$ & $4(4.76)$ \\
3 & Severe & 0 & 0 \\
4 & Extreme & 0 & 0 \\
\hline
\end{tabular}

Values are presented as number of teeth (\%).

MOPs, micro-osteoperforations.

showed mild and moderate resorption, respectively, and only 3.6\% showed severe root resorption. Overall, almost half $(47.62 \%)$ of the measured teeth showed mild root resorption and only 5.95\% showed moderate root resorption. The remaining teeth showed no incidence of severe or extreme resorption.

\section{Effects of micro-osteoperforations on external apical root resorption}

The MOP and the control groups showed no significant differences in mean root resorption $(p=0.419)$, and the changes in root length over the six-month 
period within groups were not significant respectively ( $p=0.238 \mathrm{MOP}$ and $p=0.225$ in control groups). The present study is the first to compare the EARR on the anterior maxillary teeth during initial alignment with that after MOP application. Our results are consistent with those reported by Alkebsi et al. ${ }^{21}$ for a three-month trial of MOP-assisted orthodontic canine retraction. They found that the difference in mean root resorption between the intervention and control groups, with EARRs of $0.61 \mathrm{~mm}$ and $0.73 \mathrm{~mm}$, respectively, was not significant. Similarly, the split-mouth study by Aboalnaga et al., ${ }^{22}$ also found no significant difference in EARR in the four-month canine retraction period. The latter study used adjusted cone-beam computed tomography (CBCT) for measurement of root resorption. In contrast, a microcomputed tomography (micro-CT) RCT on the extracted first maxillary premolars following 28 days of buccal tipping with a force of $150 \mathrm{~g}$ reported that MOPs significantly increased EARR by $0.17 \mathrm{~mm} .{ }^{23}$ However, the results of that study should be considered with caution, since the clinical relevance of that technique may be debatable in general clinical practice.

The lesser extent of EARR exhibited by the MOP group is in agreement with the findings of a previous trial. ${ }^{9}$ This is due to regional acceleratory phenomenon activity induced by the disruption of the alveolar bone by MOPs, which causes intensification of osteoclastic activity and reduced bone density. Such a reaction subsequently reduces the possibility of hyalinization necrosis and resorption of the tooth root. Additionally, the increased cortical bone porosity simultaneously reduces mechanical resistance for OTM and EARR. ${ }^{9}$

\section{Diagnostic tools}

Most EARR diagnoses are based on radiographic evaluation, with apical blunting or shortening of the root length appearing as the first noticeable signs. Root resorption is measured by using IOPA radiographs with a CF adapted from previous studies to compensate for image magnification. ${ }^{17,24}$ One of the limitations of using 10PA is the tendency to underestimate apical root resorption. This is justified by a study performed by Dudic et al. $^{25}$ that aimed to validate the accuracy of 10PA against a micro-CT scanner method in the diagnosis of EARR on extracted teeth. 10PA detected only 55\% of EARRs in the experimental teeth whereas the micro-CT scans detected $86 \%$ of the EARRs on similar teeth. The most reliable and valid tool to assess root resorption is CBCT. It allows the roots to be visualized and evaluated on any surface and eradicates the image superimposition. It also ensures accuracy in the measurement and ensures better comparability. Nevertheless, CBCT exposes patients with a higher dose of radiation and is more expensive. ${ }^{26}$ For these reasons, CBCT endorsement in or- thodontics is limited to specific indications.

The effectiveness of detecting EARR by using twodimensional intraoral radiographs is arguable. Root resorption is a three-dimensional process that can occur at any of the root surfaces. Our EARR evaluation used two-dimensional long-cone periapical radiographs, which could limit the accuracy of root resorption calculation and its occurrence. Even though this approach shows limitations in evaluating all surfaces and tends to underestimate root resorption, the clinical relevance of the undetected EARR on plain radiographic images is debatable. The severity of such EARR is usually clinically insignificant. Moreover, both CBCT and 1OPA have been shown to detect a comparable percentage of moderate and severe EARR. Thus, the protocol using IOPA radiographs in this study reflected contemporary orthodontic practice, yielding a meaningful trial outcome pertinent to routine clinical practice.

In general, mild to moderate EARR rarely shows clinical significance unless it is accompanied by severe or extreme resorption. However, root resorption of more than quarter of its length may adversely affect root longevity and yield an unfavorable crown-root ratio. ${ }^{27}$ Remarkably, Levander and Malmgren ${ }^{28}$ suggested that longterm tooth survival was not compromised even in cases with major EARR. Sondeijker et al. ${ }^{29}$ developed a comprehensive clinical practice guideline that recommended different management approaches for EARR before, during, and after orthodontic treatment. Even though the guidelines were established principally from a universal agreement between clinicians with limited existing evidence, they permitted clinicians to act in response to EARR based on present expertise. The guidelines include a strong recommendation to inform patients about the risk of EARR prior to orthodontic treatment, with greater caution to be exercised in extraction cases, which are associated with a greater possibility of severe EARR. They also strongly recommend reassessment and revision of the treatment aims and plan when EARR equal to or more than $2 \mathrm{~mm}$ is apparent during fixed appliance treatment. ${ }^{29}$

\section{Adverse effects}

No root perforation or soft tissue scarring were identified throughout the trial period in all participants. No accidental adverse effects were reported.

\section{Limitations}

Root resorption is a three-dimensional process that can occur at any of the root surfaces. Our EARR evaluations were performed using two-dimensional 10PA, which could limit the accurate calculation of resorption, since its occurrence is three-dimensional. Future studies should investigate the effect of MOPs throughout the 
treatment duration to assess its complete effectiveness.

The clinical effectiveness of MOPs on the rate of tooth movement over a broader range of orthodontic treatments remains to be investigated. The alignment duration and pain experienced by the patients with the additional MOPs are described in another part of the study.

\section{Generalizability}

The generalizability of this study may be limited by the single-center nature of the clinical trial. However, these findings are representative of general clinical practice, where standard mechanotherapy is performed in conventional orthodontic treatment for adults.

\section{CONCLUSION}

Within the conditions of the present RCT, MOP therapy during the orthodontic alignment phase does not exacerbate EARR when compared to the control group.

\section{CONFLICTS OF INTEREST}

No potential conflict of interest relevant to this article was reported.

\section{ACKNOWLEDGEMENTS}

We would like to express our sincere gratitude to Mrs. Izyan Hazwani Baharuddin for her expert guidance on the biostatistical analysis, as well as our colleagues for their assistance during the subject recruitment period.

\section{REFERENCES}

1. Iglesias-Linares A, Hartsfield JK Jr. Cellular and molecular pathways leading to external root resorption. J Dent Res 2017;96:145-52.

2. Fahey T, Stephenson P. Root resorption during fixed appliance therapy- mechanism, risk factors and prognosis. Orthod Update 2016;9:96-9.

3. Nowrin SA, Jaafar S, Ab Rahman N, Basri R, Alam MK, Shahid F. Association between genetic polymorphisms and external apical root resorption: a systematic review and meta-analysis. Korean J Orthod 2018;48:395-404.

4. Roscoe MG, Meira JB, Cattaneo PM. Association of orthodontic force system and root resorption: a systematic review. Am J Orthod Dentofacial Orthop 2015;147:610-26.

5. Weiland F. Constant versus dissipating forces in orthodontics: the effect on initial tooth movement and root resorption. Eur J Orthod 2003;25:335-42.

6. Kalemaj Z, Debernardl CL, Buti J. Efficacy of surgical and non-surgical interventions on accelerating orthodontic tooth movement: a systematic review. Eur J Oral Implantol 2015;8:9-24.

7. Al-Naoum F, Hajeer MY, Al-Jundi A. Does alveolar corticotomy accelerate orthodontic tooth movement when retracting upper canines? A split-mouth design randomized controlled trial. J Oral Maxillofac Surg 2014;72:1880-9.

8. Gkantidis N, Mistakidis I, Kouskoura T, Pandis N. Effectiveness of non-conventional methods for accelerated orthodontic tooth movement: a systematic review and meta-analysis. J Dent 2014;42:1300-19.

9. Abbas NH, Sabet NE, Hassan IT. Evaluation of corticotomy-facilitated orthodontics and piezocision in rapid canine retraction. Am J Orthod Dentofacial Orthop 2016;149:473-80.

10. Charavet C, Lecloux G, Jackers N, Maes N, Lambert F. Patient-reported outcomes measures (PROMs) following a piezocision-assisted versus conventional orthodontic treatments: a randomized controlled trial in adults. Clin Oral Investig 2019;23:4355-63.

11. Charavet C, Lecloux G, Bruwier A, Rompen E, Maes $\mathrm{N}$, Limme $\mathrm{M}$, et al. Localized piezoelectric alveolar decortication for orthodontic treatment in adults: a randomized controlled trial. J Dent Res 2016; 95:1003-9.

12. Charavet C, Van Hede D, Anania S, Maes N, Lambert F. Multilevel biological responses following piezocision to accelerate orthodontic tooth movement: a study in rats. J World Fed Orthod 2019;8:100-6.

13. Charavet C, Lecloux G, Jackers N, Albert A, Lambert F. Piezocision-assisted orthodontic treatment using CAD/CAM customized orthodontic appliances: a randomized controlled trial in adults. Eur J Orthod 2019;41:495-501.

14. Sivarajan S, Ringgingon LP, Fayed MMS, Wey MC. The effect of micro-osteoperforations on the rate of orthodontic tooth movement: a systematic review and meta-analysis. Am J Orthod Dentofacial Orthop 2020;157:290-304.

15. Alikhani M, Raptis M, Zoldan B, Sangsuwon C, Lee YB, Alyami B, et al. Effect of micro-osteoperforations on the rate of tooth movement. Am J Orthod Dentofacial Orthop 2013;144:639-48.

16. Nanekrungsan K, Patanaporn V, Janhom A, Korwanich N. External apical root resorption in maxillary incisors in orthodontic patients: associated factors and radiographic evaluation. Imaging Sci Dent 2012;42:147-54.

17. DiBiase AT, Woodhouse NR, Papageorgiou SN, Johnson N, Slipper C, Grant J, et al. Effect of supplemental vibrational force on orthodontically induced inflammatory root resorption: a multicenter randomized clinical trial. Am J Orthod Dentofacial Orthop 2016;150:918-27. 
18. Levander E, Malmgren 0. Evaluation of the risk of root resorption during orthodontic treatment: a study of upper incisors. Eur J Orthod 1988;10:30-8.

19. Kocadereli 1, Yesil TN, Veske PS, Uysal S. Apical root resorption: a prospective radiographic study of maxillary incisors. Eur J Dent 2011;5:318-23.

20. Smale 1, Artun J, Behbehani F, Doppel D, van't Hof M, Kuijpers-Jagtman AM. Apical root resorption 6 months after initiation of fixed orthodontic appliance therapy. Am J Orthod Dentofacial Orthop 2005;128:57-67.

21. Alkebsi A, Al-Maaitah E, Al-Shorman H, Abu Alhaija E. Three-dimensional assessment of the effect of micro-osteoperforations on the rate of tooth movement during canine retraction in adults with Class 11 malocclusion: a randomized controlled clinical trial. Am J Orthod Dentofacial Orthop 2018;153:771-85.

22. Aboalnaga AA, Salah Fayed MM, El-Ashmawi NA, Soliman SA. Effect of micro-osteoperforation on the rate of canine retraction: a split-mouth randomized controlled trial. Prog Orthod 2019;20:21.

23. Chan E, Dalci O, Petocz P, Papadopoulou AK, Darendeliler MA. Physical properties of root cementum: part 26. Effects of micro-osteoperforations on orthodontic root resorption: a microcomputed tomography study. Am J Orthod Dentofacial Orthop 2018;153:204-13.
24. Motokawa M, Sasamoto T, Kaku M, Kawata T, Matsuda Y, Terao A, et al. Association between root resorption incident to orthodontic treatment and treatment factors. Eur J Orthod 2012;34:350-6.

25. Dudic A, Giannopoulou C, Martinez M, Montet X, Kiliaridis S. Diagnostic accuracy of digitized periapical radiographs validated against micro-computed tomography scanning in evaluating orthodontically induced apical root resorption. Eur J Oral Sci 2008;116:467-72.

26. Signorelli L, Patcas R, Peltomäki T, Schätzle M. Radiation dose of cone-beam computed tomography compared to conventional radiographs in orthodontics. J Orofac Orthop 2016;77:9-15.

27. Janson GR, De Luca Canto G, Martins DR, Henriques JF, De Freitas MR. A radiographic comparison of apical root resorption after orthodontic treatment with 3 different fixed appliance techniques. Am J Orthod Dentofacial Orthop 2000;118:262-73.

28. Levander E, Malmgren 0. Long-term follow-up of maxillary incisors with severe apical root resorption. Eur J Orthod 2000;22:85-92.

29. Sondeijker CFW, Lamberts AA, Beckmann SH, Kuitert $\mathrm{RB}$, van Westing K, Persoon S, et al. Development of a clinical practice guideline for orthodontically induced external apical root resorption. Eur J Orthod 2020;42:115-24. 